\title{
An alternative projection for fluoroscopic-guided needle insertion in the foramen ovale: technical note
}

\author{
Peter Grunert • Joachim Oertel
}

Received: 2 August 2010/Accepted: 4 August 2010/Published online: 18 November 2010

(C) Springer-Verlag 2010

Dear Prof. De Tribolet:

We thank Drs. Arrese and Urculo for their careful considerations of our publication on fluoroscopic-guided needle insertion in the foramen ovale. The authors describe an alternative procedure to control the needle position in the foramen ovale. They apply neuronavigation by inserting a magnetic guide inside the needle as a pointer. We congratulate the authors for their splendid idea and for the nice technique they developed. Never having done such a procedure, the technique appears to be elegant and adequate. There are always different approaches to a single problem. To us, the most important issue is that the needle position in the foramen ovale can be controlled with a simple and almost always readily available technique without significant additional costs. Thus, to us, application of neuronavigation seems to be rather oversized having an online technique available which gives us realtime information on the needle position as described in our publication. However, further studies might define specific indications for any of both techniques.

With best regards.

Sincerely,

Peter Grunert, MD

Joachim Oertel, MD

Conflicts of interest None.

P. Grunert · J. Oertel

Klinik für Neurochirurgie,

Universitaetsmedizin, Johannes Gutenberg-Universitaet,

Mainz, Germany

J. Oertel $(\bowtie)$

Neurochirurgische Klinik und Poliklinik, Universitaetsmedizin, Johannes Gutenberg Universität,

Langenbeckstrasse 1,

55131 Mainz, Germany

e-mail: oertel@nc.klinik.uni-mainz.de 